\title{
Tamanho e Número Ideal de Amostras para Coleta de Gafanhotos na Região Depressão Central do Rio Grande do Sul
}

\author{
Nathália Leal Carvalho1 ${ }^{\bowtie}$, Ervandil Correa Costaㅜ, Danilo Boanerges Souza² \& Juliana Garlet²
}

1.Universidade de Passo Fundo, e-mail: nathaliiinha@gmail.com (Autor para correspondência ${ }^{\bowtie}$ ). 2. Universidade Federal de Santa Maria, e-mail: eccosta@gmail.com, daniloboanerges@gmail.com, julianagatlet@yahoo.com.br.

EntomoBrasilis 6(2): 119-125 (2013)

Resumo. Objetivando observar a viabilidade de um novo método para amostragem de acridóideos (conhecidos vulgarmente por gafanhotos), bem como a distancia percorrida e número ideal de amostras para amostragem foi proposto o estudo. Os levantamentos foram conduzidos em duas áreas de campo nativo no município de São Sepé, localizado na região Depressão Central no RS. As coletas foram realizadas durante o verão de 2009 com o auxilio de uma rede de coleta adaptada. As distâncias avaliadas foram de 5, 10, 15, 20 e 25m, com oito repetições cada, sendo 40 coletas por área, a amostragem foi ao acaso. As variáveis respostas foram: adultos, ninfas, espécies e número total de insetos. Foram calculadas as estatísticas descritivas, o teste Qui-quadrado a 5\% de probabilidade de erro e à relação mediana/pseudo-sigma, aliada a metodologia de reamostragem Jackknife. Foram coletados 969 espécimes distribuídos em 22 espécies, 17 Gêneros, três Famílias distintas Acrididae, Proscopiidae e Romaleidae, e duas Superfamílias (Acridoidea e Eumastacoidea). Evidenciou-se relativa eficiência do método proposto. A distancia percorrida de $25 \mathrm{~m}$ aliada ao número de sete amostras mostrou-se ideal para coleta de acridóideos.

Palavras-chave: Acridóideos; Biodiversidade; Entomofauna; Orthoptera.

\section{Ideal Size and Number of Samples to Collect Grasshoppers in the Central Depression in Rio Grande do Sul}

Abstract. Aiming to observe the viability of a new method for sampling acridoideos (by commonly known locusts) and the ideal distance and number of samples for the study sample was proposed. The surveys were conducted in two areas of native grassland in Sao Sepe, in Central Depression, Rio Grande do Sul State. Samples were collected during the summer of 2009 with the help of a network of collection adapted. The distances were measured 5, 10, 15, 20 and 25m, with eight repetitions each, of which 40 samples per area, sampling was random. The response variables were: adults, nymphs, and total number of species of insects. Descriptive statistics were calculated, Chi-square test and used the median ratio/pseudosigma methodology combined with Jackknife resampling. We collected 969 specimens representing 22 species, 17 Genres and three distinct families Acrididae, Proscopiidae and Romaleidae, and also two Superfamilies (Acridoidea and Eumastacoidea). It was evident relative efficiency of the proposed method. The distance traveled of $25 \mathrm{~m}$ coupled with the number seven samples proved to be ideal for collecting of grasshoppers.

Keywords: Acridoideos; Biodiversity; Entomofauna; Orthoptera.

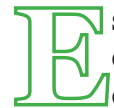
studos envolvendo amostragens de insetos são efetuados com o objetivo de conhecer e identificar a entomofauna de um determinado ecossistema, bem como a densidade ou o número de espécies que ocupam esta área, a dispersão ou o arranjo dos indivíduos no espaço, mudanças nas taxas de natalidade e mortalidade, número relativo de vários estágios dos insetos e mudanças em seu número, ao longo do tempo (SubRAMANYAM \& HagSTRum 1996).

O conhecimento de uma forma de amostragem rápida e eficiente de insetos é fundamental, principalmente de espécies-praga e seus inimigos naturais, principalmente quando se trabalha em Programas de Manejo Integrado de Pragas (Fernandez et al. 2002). Pois é a partir da amostragem que se determinam os parâmetros quantitativos e qualitativos das diferentes espéciespraga, detectando a presença do inseto em determinada cultura, e sua abundância no tempo e no espaço. Estas informações podem ser usadas para predizer tendências populacionais no futuro e avaliar danos potenciais (PEDIGO 1994).

Para um programa de monitoramento, vários fatores devem ser considerados, dentre eles a localização, o número e tamanho das amostras a serem coletadas, e a frequência de amostragem. Esses fatores não são independentes. A amostragem pode ser menos frequente se um grande número de amostras for tomado, pois haverá maior confiança de que não se esteja subestimando a densidade dos insetos e de que os níveis de densidade populacional não terão atingido o limite crítico (HaGSTRum et al. 1994).

A estimativa da população de insetos em determinada área ou ecossistema, torna-se essencial para vários fins, principalmente, quando se deseja determinar limites populacionais, para a utilização de métodos eficazes de controle (Subramanyan \& Harein 1996). A relação entre o número médio de insetos por amostra e a variância entre as amostras ou porcentagem de amostras com insetos, fornecem duas medidas de uniformidade da distribuição dos dados de densidade dos insetos em determinada área.

No entanto, trabalhos abordando número e tamanho ideal de amostras são mais frequentes em insetos-praga que causam danos a culturas agrícolas, como os associados à soja, milho, 
T algodão entre outras. Os ortópteros em geral são pouco estudados, no entanto, este grupo apresenta diversos representantes considerados pragas importantes em vários agroecossistemas, desde florestas, culturas agrícolas e pastagens. Assim estudos sobre métodos de coleta, bem como, tamanho e número ideal de amostras, para avaliação deste grupo em diferentes ecossistemas se torna importante, não somente pela importância econômica, como ambiental, que estes insetos representam.

Assim, o objetivo deste estudo foi observar a viabilidade de um novo método para amostragem de acridoídeos, bem como a distancia percorrida e número ideal de amostras para amostragem de insetos deste grupo.

\section{MATERIAL E MÉTODOS}

Os levantamentos foram realizados em duas áreas na localidade de São Rafael, município de São Sepé, na região Depressão Central do Rio Grande do Sul, Brasil ( $30^{\circ}$ o9' $38^{\prime}$ ' S e $53^{\circ} 33^{\prime}$ 55 ” W), estando a uma altitude de 85 metros. A primeira área (Área A) possui 82 ha, é caracterizada por campo nativo onde foi cultivado trigo, por várias safras. A segunda área (Área B) possui 92 ha de campo nativo, onde não houve cultivo algum. As áreas encontram-se distantes uma da outra em $3 \mathrm{~km}$ no sentido lesteoeste (A para B), sendo estas circundadas por lavouras de soja e campo nativo.

O Clima da região, conforme a classificação de Köppen é Cfa, correspondendo a Subtropical Temperado Úmido. Tem como principais características temperaturas médias anuais em torno de $17^{\circ} \mathrm{C}$, e com uma amplitude térmica superior a $10^{\circ} \mathrm{C}$ entre o dia e a noite. A umidade relativa do ar é de $76 \%$ em média, e o regime de chuvas ao redor de $1500 \mathrm{~mm} / \mathrm{ano}$, sendo que a maior parte é concentrada no outono e inverno (MORENo 1961).

Para a realização das amostragens, foi utilizada uma rede de coleta, que consiste em um aro de ferro de $0,005 \mathrm{~m}$ com $1,5 \mathrm{~m}$ de comprimento por $1,3 \mathrm{~m}$ de altura, com duas dobradiças no meio, onde duas pessoas seguram a rede na parte superior, aproximadamente na linha dos ombros, quase encostando no solo, percorrendo aleatoriamente no campo certa distância (Figura 1). Após o caminhamento, fecha-se a rede conseguindo assim capturar os exemplares. Foram avaliadas cinco distâncias de caminhamento, sendo 5, 10, 15, 20 e $25 \mathrm{~m}$, com oito repetições cada, sendo 40 coletas em cada área, totalizando 80 amostras, a fim de verificar qual a melhor distância a ser percorrida para a coleta de ortópteros. A amostragem foi ao acaso, e após o caminhamento aleatório, e em cada distância, foram retiradas aleatoriamente por sorteio oito amostras.

Os insetos capturados foram acondicionados em sacos plásticos contendo um chumaço de algodão com éter e levados ao Laboratório de Entomologia Florestal do Departamento de Defesa Fitossanitária da Universidade Federal de Santa Maria (UFSM), RS, para separação, contagem e montagem. Após a triagem os espécimes foram encaminhados ao Laboratório de Entomologia do Museu de Ciência e Tecnologia da Pontifícia Universidade Católica do Rio Grande do Sul (PUCRS), onde foram identificados até a categoria taxonômica de espécie pela Prof ${ }^{\mathrm{a}}$ Dra $^{\mathrm{a}}$. Maria Kátia Matiotti da Costa. Os exemplares identificados se encontram depositados na coleção do Museu de Ciências e Tecnologia da PUC/RS do setor de Entomologia.

As variáveis avaliadas foram: número de insetos adultos, número de ninfas, número de espécies e número total de insetos.

Utilizando-se o teste de Lilliefors (5\%), verificou-se que os resíduos da variável não seguiam distribuição normal. Assim, foram testados diversos tipos de transformações de dados, com as quais não foi possível obter normalidade. Dessa forma, optou-se por analisar os dados através do teste não paramétrico de distribuição livre, Qui-quadrado, para independência ou associação, em nível de $5 \%$ de probabilidade de erro, no qual foram testadas as hipóteses: Ho - o número de indivíduos em cada umas das espécies independe das áreas (“A” e "B”); e H1 - o número de indivíduos em cada umas das espécies depende das áreas ("A" e "B"). O objetivo de aplicação desse teste foi para verificar a necessidade de calcular os índices de diversidade para cada área, ou para o conjunto de dados das duas áreas. O procedimento de cálculo para realização do teste Qui-quadrado para independência ou associação está descrito em Fonseca \& Martins (2009). Chegando a conclusão que áreas podem ser analisadas conjuntamente.
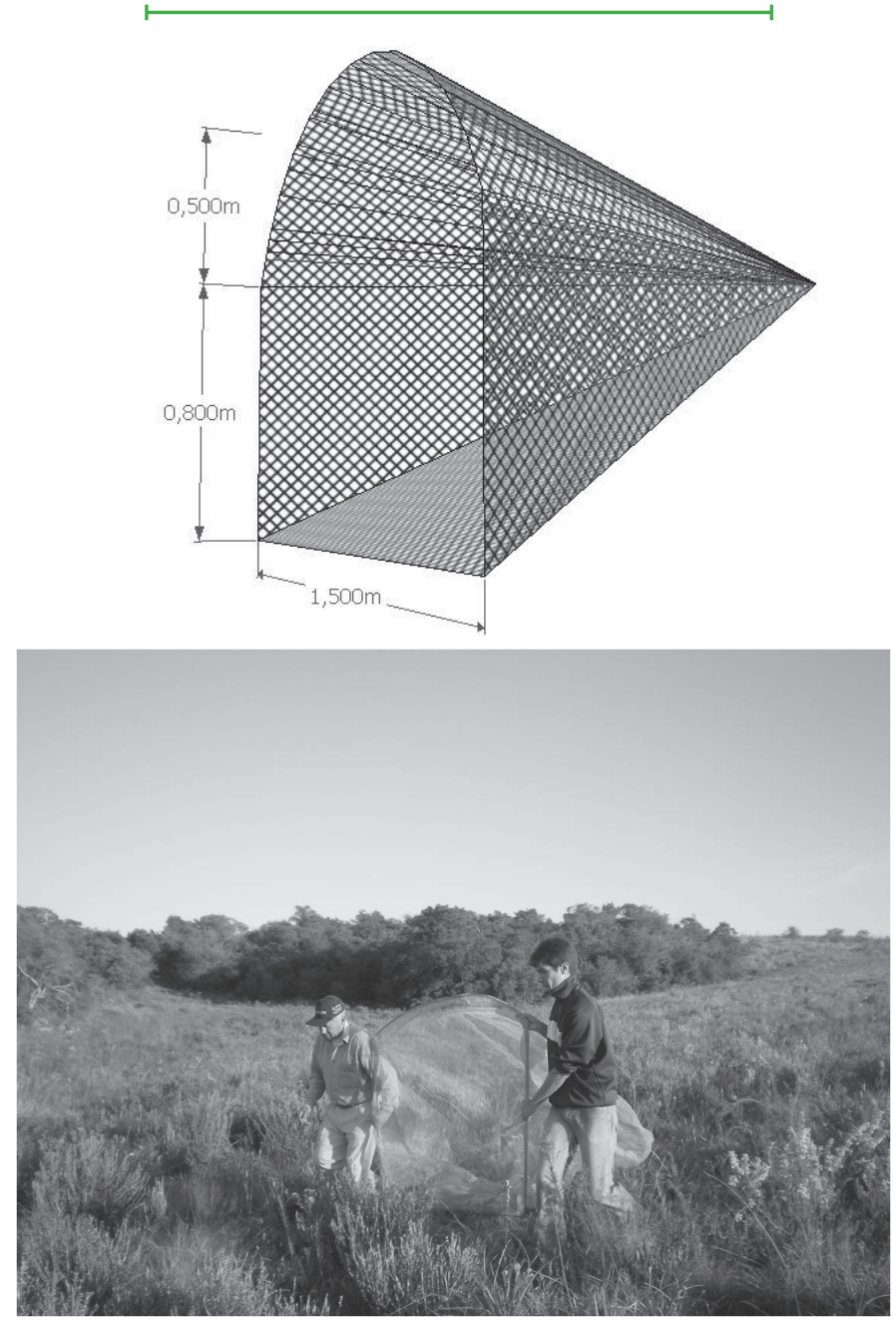

Figura 1. Ilustração e coleta de acridóideos com rede de coleta modificada, proposta por CARVALHO \& CoSTA (2010). São Sepé, RS, 2009.

Foram calculadas as estatísticas descritivas e para análise dos dados foi utilizado à relação mediana/pseudo-sigma, aliada a metodologia de reamostragem Jackknife (MANLY 1997; CONFALONIERI 2004).

\section{RESULTADOS E DISCUSSÃO}

Durante este levantamento, foram coletados 969 espécimes distribuídos em 22 espécies, 17 Gêneros, três Famílias distintas Acrididae, Proscopiidae e Romaleidae, e duas Superfamílias Acridoidea e Eumastacoidea, apresentados na Tabela 1.

Pelos dados da Tabela 1, observa-se que a família Acrididae, apresentou o maior número de espécies e indivíduos coletados em ambas as áreas, em relação a família Proscopiidae Foi encontrado apenas um indivíduo da espécie Oriencospia angustirostris (Bruner) e o número de indivíduos não variou em ambas as áreas. Na área "A" destacaram-se as espécies Scotussa cliens Stål com 136 indivíduos coletados, seguido de Dichroplus silveiraguidoi Liebermann com 112 exemplares, que também se destacou na área "B" com 86 indivíduos coletados. As espécies Cylindrotettix chacoensis Roberts e Dichroplus conspersus Bruner apresentaram o mesmo número de exemplares em ambas as áreas (três e quatro, respectivamente). 
Tabela 1- Número de acridóideos coletados por família e espécie em São Sepé, RS, 2009.

\begin{tabular}{|c|c|c|c|}
\hline Superfamília & Espécie/Família & $\begin{array}{c}\text { Número de } \\
\text { Indivíduos área " } A \text { " }\end{array}$ & $\begin{array}{c}\text { Número de } \\
\text { Indivíduos área "B" }\end{array}$ \\
\hline \multirow[t]{23}{*}{ Acridoidea } & Acrididae & & \\
\hline & Aleuas lineatus Stål & 3 & 10 \\
\hline & Aleuas gracilis Stål & 2 & 6 \\
\hline & Aleuas viticollis Stål & 19 & 26 \\
\hline & Allotruxalis strigata (Bruner) & 9 & 11 \\
\hline & Ambytropidia robusta Bruner & 7 & 9 \\
\hline & Ambytropidia sola Rehn & 20 & 27 \\
\hline & Borellia bruneri (Rehn) & 4 & 6 \\
\hline & Borellia pallida (Bruner) & 3 & 3 \\
\hline & Cylindrotettix chacoensis Roberts & 4 & 4 \\
\hline & Dichroplus conspersus Bruner & 19 & 42 \\
\hline & Dichroplus silveiraguidoi Liebermann & 112 & 89 \\
\hline & Fenestra bohlsii Giglio-Tos & 15 & 19 \\
\hline & Notopomala glaucipes Rehn & 61 & 34 \\
\hline & Orphulella punctata (De Geer) & 2 & 13 \\
\hline & Parorphula graminea Bruner & 12 & 9 \\
\hline & Rhammatocerus pictus (Bruner) & 17 & 32 \\
\hline & Scotussa cliens Stål & 136 & 57 \\
\hline & Staurorhectus longicornis longicornis Giglio-Tos & 35 & 26 \\
\hline & Romaleidae & & \\
\hline & Staleochlora arcuata iguazuensis Roberts \& Carbonell & 5 & 10 \\
\hline & Xyleus laevipes (Stål) & 4 & 6 \\
\hline & Zoniopoda tarsata (Serville) & 5 & 16 \\
\hline \multirow[t]{2}{*}{ Eumastacoidea } & Proscopiidae & & \\
\hline & Oriencospia angustirostris (Bruner) & 10 & 10 \\
\hline Total & & 504 & 465 \\
\hline
\end{tabular}

Através do teste não paramétrico de distribuição livre, Quiquadrado, para independência ou associação, concluiu se que o teste foi significativo para as variáveis número de insetos adultos, número de espécies e número total de insetos, e mostra que o mais indicado é analisar conjuntamente as duas áreas, exceto para a variável número de ninfas. Tal fato pode ter ocorrido devido à variação das plantas existentes em cada área, as quais servem de alimento e refúgio das espécies, ao uso do solo e das condições climáticas e microclima da região no momento da captura. O gráfico de distribuição dos exemplares coletados nas distancias analisadas foi confeccionado (Figura 2).

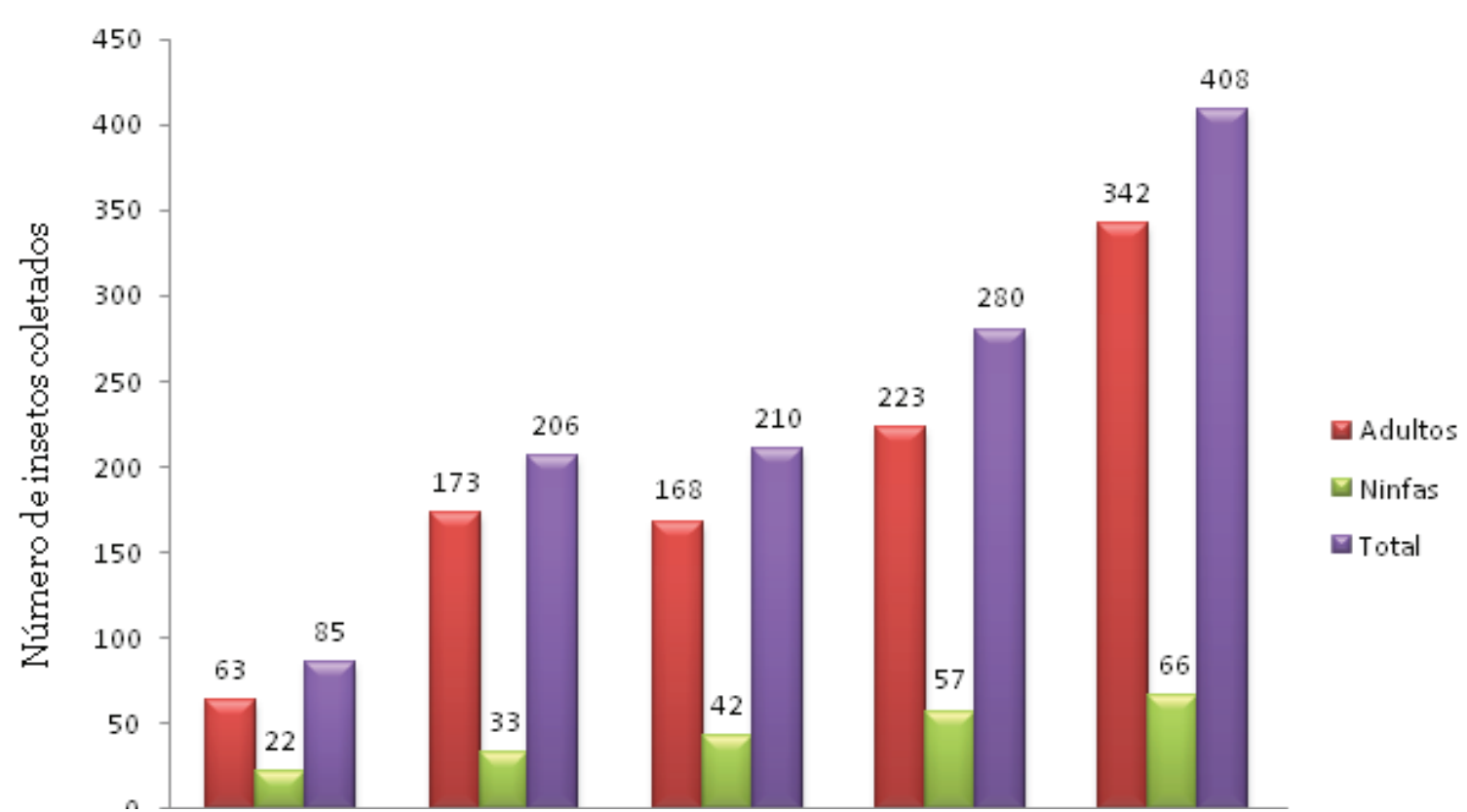

Figura 2. Número de insetos coletados em cada tamanho de amostra (distância percorrida), em ambas as áreas, São Sepé, RS, 2009. 
T Pelos dados da Figura 2, constata-se que foram coletados mais adultos (969) do que ninfas (220) nas cinco distancias avaliadas, em ambas as áreas, e o aumento da distancia percorrida durante a amostragem proporcionou acréscimo do número de indivíduos coletados. Houve um acréscimo no número de insetos adultos, ninfas e total em ambas as áreas, com o aumento da distância percorrida. Em todas as distâncias, houve maior quantidade de indivíduos adultos em relação a ninfas. Com o aumento do tamanho da amostra, percebe-se que o aumento de exemplares capturados não foi proporcional, mas houve um acréscimo significativo na diversidade das espécies coletadas.

Observa-se no estudo que, o número de espécies coletadas aumentou com o acréscimo do tamanho da amostra, com exceção da distancia de $15 \mathrm{~m}$, em que se observou menor número de espécies do que na amostra de 10m. Pela Figura 3 constata-se também que com o aumento do tamanho de amostra de 5 para $25 \mathrm{~m}$ (diferença de $20 \mathrm{~m}$ ), obteve-se um acréscimo de quatro vezes no número de espécies de acridóideos coletados, ou seja, a distância de $25 \mathrm{~m}$ apresentou uma diversidade de espécies significativamente maior que as distancias de 5, 10 e $15 \mathrm{~m}$ (Figura $3)$.
Ao agrupar as amostras das áreas "A" e "B", os valores médios são iguais a 12, 15 e seis, para as variáveis número de insetos adultos, número total de insetos e número de espécies, respectivamente. A média do número de ninfas nas áreas " $\mathrm{A}$ " $\mathrm{e}$ " $\mathrm{B}$ " foi dois e quatro, respectivamente (Tabela 2 ).

Em relação às medidas de dispersão, verificam-se valores médios a elevados de coeficiente de determinação, que variam de 32,54\% (número de insetos adultos) a 105,93\% (número de ninfas). Esses valores mostram uma variabilidade maior quando se consideram as áreas separadas, como é caso da variável número de ninfas.

A amplitude de variação dos dados para as variáveis: adultos, ninfas e espécies, foi igual ao valor máximo, pois o valor mínimo observado foi igual a o (zero). O valor do primeiro quartil mostra que $25 \%$ dos valores encontram-se abaixo de seis, dois, oito e quatro, para as variáveis: Adultos, Ninfas na área "B", Total e Espécies, respectivamente.

Considerando a mediana, verifica-se que $50 \%$ dos valores encontram-se distribuídos em cada lado de: 10,5, um, quatro, 13,5 e seis, para as variáveis: adultos, ninfas na área "A", ninfas na área "B", total e espécies, respectivamente.

Tabela 2. Estatísticas descritivas das variáveis: adultos (número de insetos adultos) total (número total de insetos), ninfas (número de ninfas) e espécies (número de espécies), do conjunto de 80 amostras coletadas nas áreas "A" e "B". São Sepé, RS, 2009.

\begin{tabular}{|c|c|c|c|c|c|}
\hline \multirow[b]{2}{*}{ Estatísticas } & \multicolumn{5}{|c|}{ Variáveis } \\
\hline & $\begin{array}{c}\text { Ninfas Área } \\
\text { "A" }\end{array}$ & $\begin{array}{c}\text { Ninfas Área } \\
\text { "B" }\end{array}$ & Adultos & Total & Espécies \\
\hline Média Aritmética & 2,00 & 4,00 & 12,00 & 15,00 & 6,00 \\
\hline Tamanho da amostra & 40,00 & 40,00 & 80,00 & 80,00 & 80,00 \\
\hline Mínimo & 0,00 & 0,00 & 0,00 & 1,00 & 0,00 \\
\hline Máximo & 9,00 & 11,00 & 33,00 & 39,00 & 17,00 \\
\hline Amplitude total & 9,00 & 11,00 & 33,00 & 38,00 & 17,00 \\
\hline Mediana & 1,00 & 4,00 & 10,50 & 13,50 & 6,00 \\
\hline Primeiro Quartil (25\%) & 0,00 & 2,00 & 6,00 & 8,00 & 4,00 \\
\hline Terceiro Quartil (75\%) & 2,25 & 5,00 & 18,25 & 20,25 & 8,00 \\
\hline Variância & 3,15 & 6,81 & 0,11 & 1,48 & 0,01 \\
\hline Coeficiente de Variação & $105,93 \%$ & $68,25 \%$ & $32,54 \%$ & $33,21 \%$ & $29,82 \%$ \\
\hline
\end{tabular}

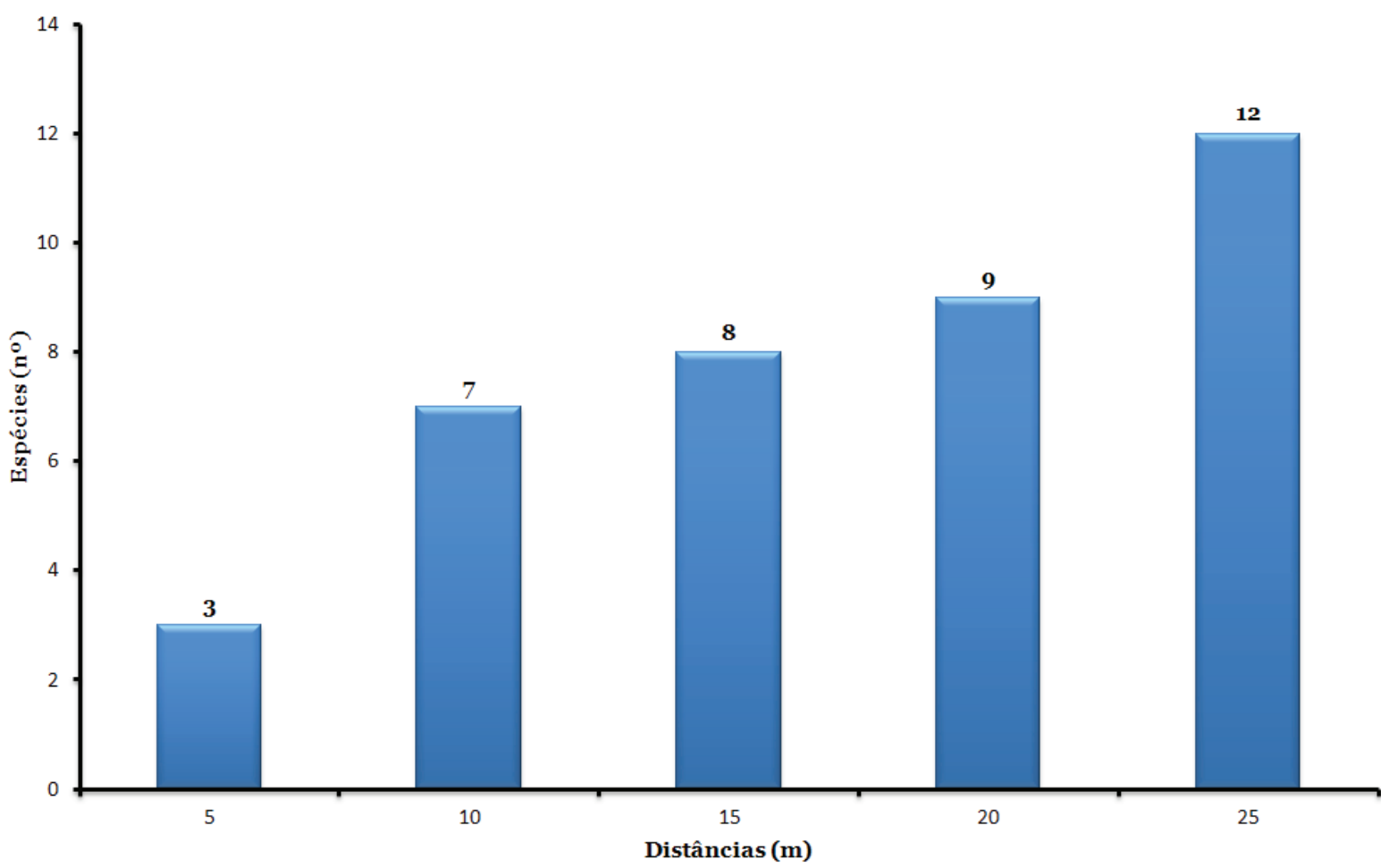

Figura 3. Número de espécies coletadas por distância percorrida em ambas as áreas, São Sepé, RS, 2009. 
Os valores do terceiro quartil variaram de 2,25, para a variável Ninfas na área "A"; a 20,25, para a variável total (Tabela 2 ).

A fim de determinar o tamanho (distancia percorrida) ideal de amostras para coleta de gafanhotos, foi realizado a relação mediana/pseudo-sigma, conforme apresentado na Tabela 3.
De acordo com Tabela 3, o tamanho ideal de amostra (distância ideal que deve ser percorrida) para a coleta de insetos adultos, número de espécies e número total de insetos de acridóideos, é de $25 \mathrm{~m}$, pois esse tamanho de amostra (distância percorrida) foi a que apresentou a maior relação mediana/pseudo-sigma (3,66 para a variável número de insetos adultos; 4,36 para a

Tabela 3. Relação mediana/pseudo-sigma para cálculo do tamanho ideal de amostra (distância a ser percorrida) para a coleta de insetos de acridóideos. São Sepé, RS, 2009.

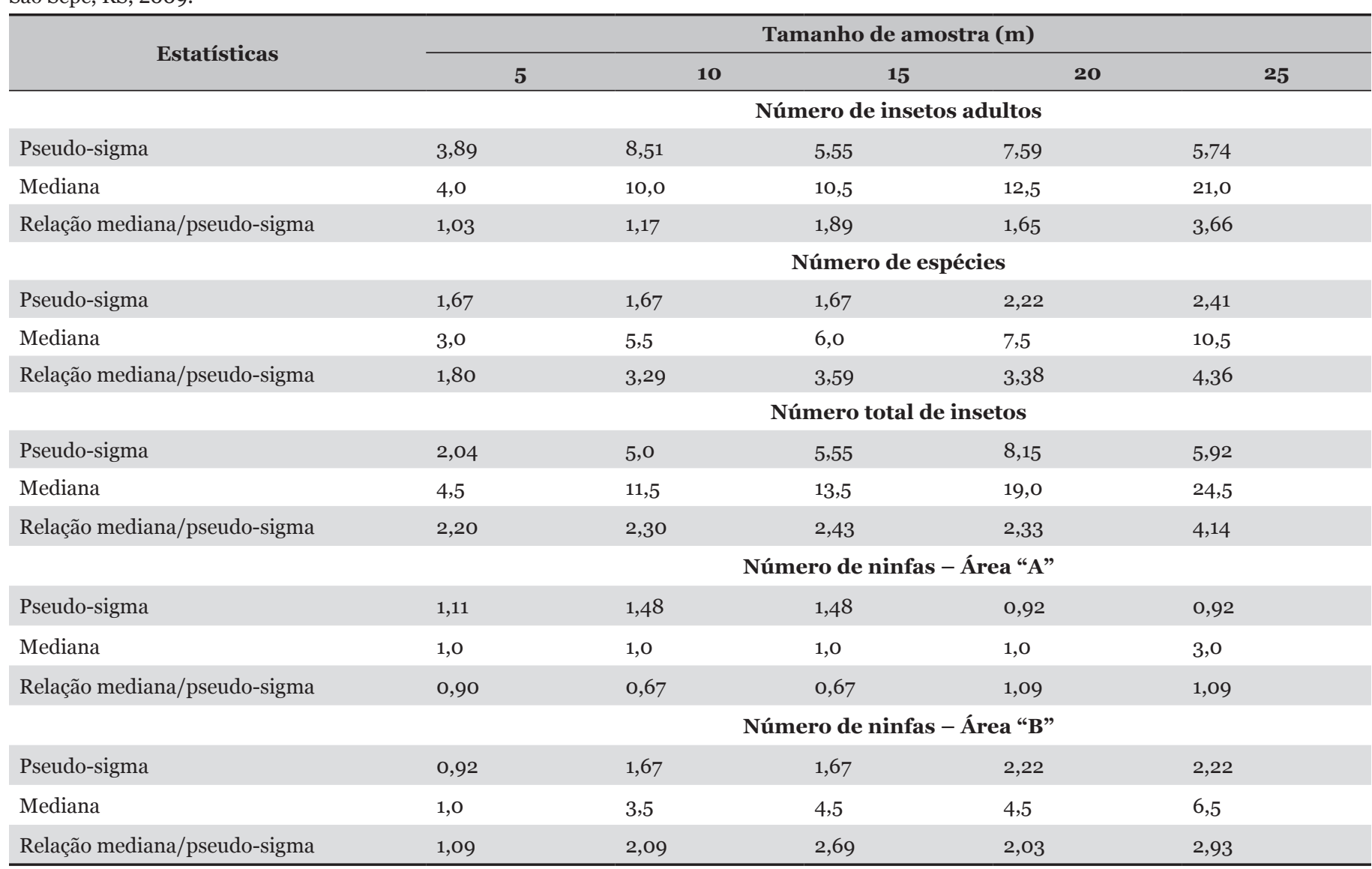

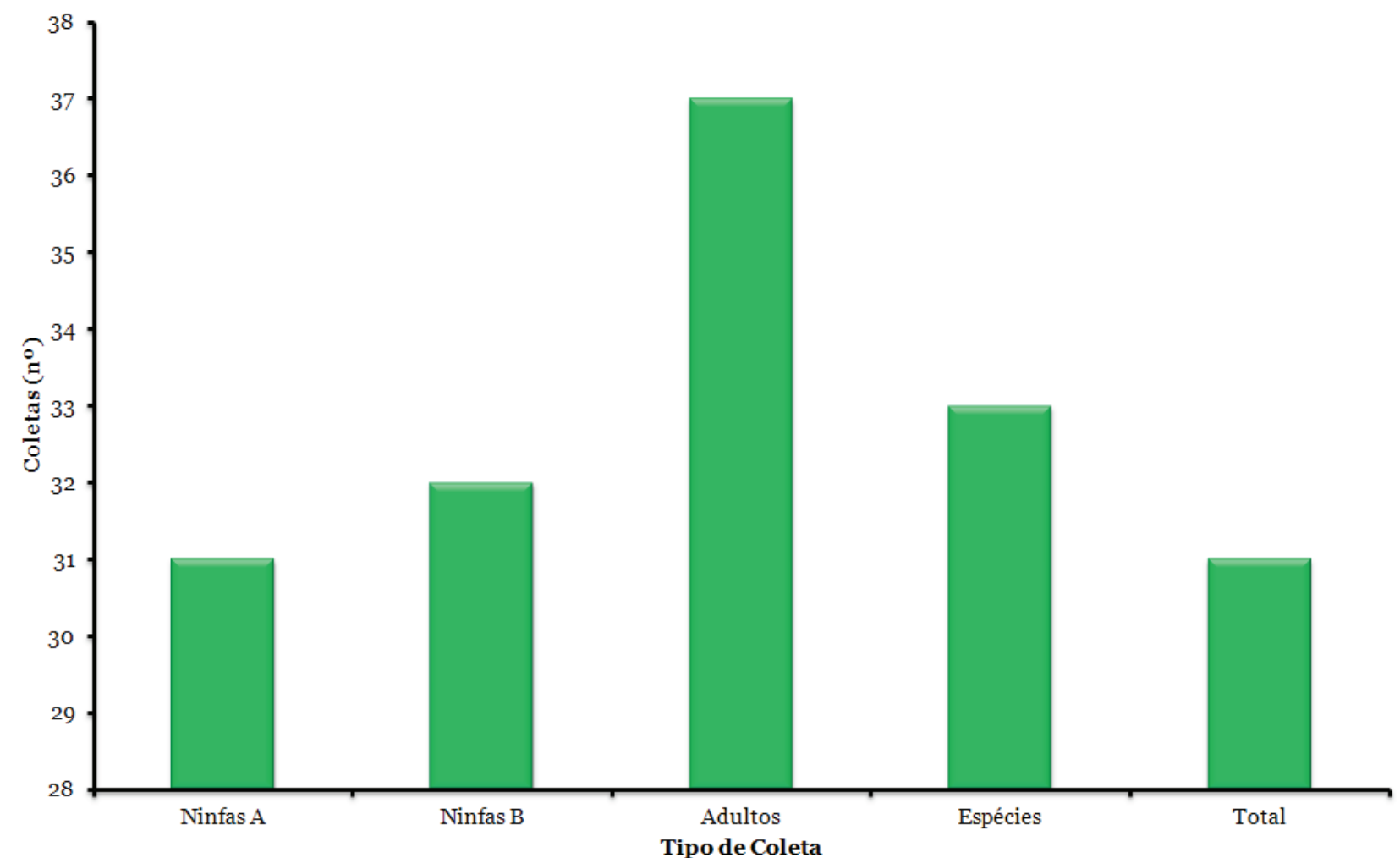

Figura 4. Número ideal de coletas para as variáveis: número de insetos adultos número de ninfas, número de espécies, e número total de insetos. São Sepé, RS, 2009. 
Tabela 4. Número ideal de amostras em cada tamanho de amostra (distância percorrida) para as variáveis: número de ninfas na área "A”, número de ninfas na área "B”, número de insetos adultos, número total de insetos e número total de espécies. São Sepé, RS, 2009.

\begin{tabular}{lccccc}
\hline $\begin{array}{c}\text { Tamanho de amostra } \\
(\mathbf{m})\end{array}$ & $\begin{array}{c}\text { Número ninfas } \\
\text { “A” }\end{array}$ & $\begin{array}{c}\text { Número ninfas } \\
\text { “B” }\end{array}$ & $\begin{array}{c}\text { Variáveis } \\
\text { Número } \\
\text { adultos }\end{array}$ & $\begin{array}{c}\text { Número } \\
\text { insetos }\end{array}$ & $\begin{array}{c}\text { Número } \\
\text { espécies }\end{array}$ \\
\hline 5 & 61 & 5 & 5 & 5 & 5 \\
\hline 10 & 6 & 6 & 5 & 7 & 6 \\
\hline 15 & 7 & 7 & 6 & 5 & 5 \\
\hline 20 & 5 & 6 & 7 & 5 & 6 \\
\hline 5 & 5 & 5 & 5 & 7 & 7 \\
\hline
\end{tabular}

${ }^{1}$ Número de amostras.

variável número de espécies e 4,14 para a variável número total de insetos).

Para a variável número de ninfas na área "A", o tamanho de amostra (distâncias percorridas) de 20 e $25 \mathrm{~m}$ apresentou valores iguais e também os maiores valores da relação mediana/pseudosigma. Assim, ambas podem ser indicadas como tamanho (distância percorrida) ideais que devem ser seguidos para a coleta de acridóideos. Já para área "B", o tamanho de amostra ideal a ser seguido para a coleta dessa variável foi $25 \mathrm{~m}$ (relação mediana/pseudo-sigma $=2,93$ ).

Portanto, recomenda-se percorrer $25 \mathrm{~m}$ com a rede de varredura para a coleta de acridóideos, pois esse tamanho de amostra (distância percorrida) permite a coleta de maior número de insetos associada à redução na variabilidade dos dados amostrais. Na determinação do número ideal de coletas, foi utilizada a metodologia de reamostragem Jackknife (MANLY 1997; CONFALONIERI 2004), considerando o total de 80 amostras, é de 37; 31; 33 e 31 para as variáveis número de insetos adultos, número de espécies, número de ninfas e número total de insetos, respectivamente. Portanto, o número ideal de coletas deve ser definido com base na variável resposta que apresentou maior número, o qual é igual a 37, conforme a figura 4.

Observa-se que o número ideal de repetições, em cada uma das distâncias percorridas, é sete. Esse número amostral foi escolhido em quase todos os tamanhos de amostras analisados: 10, 15, 20 e $25 \mathrm{~m}$; sendo que o número máximo de repetições foi sete e o mínimo cinco (Tabela 4).

O tamanho de amostra (distância percorrida) de $5 \mathrm{~m}$ foi o que apresentou menores números de amostras, em praticamente todas as variáveis, com exceção do número de ninfas. Esse resultado se deve a menor variabilidade existente entre as amostras ao se percorrer distâncias menores.

Richman et al. (1993) citam que o método de captura rede de varredura é muito eficiente na amostragem de gafanhotos. Porém, existem espécies que mesmo abundantes no ambiente raramente são capturadas por este método. LUTINSKI et al. (2009), avaliando a fauna de gafanhotos em plantios de Eucalyptus spp. e Pinus elliottii Engelm, coletaram um total de 2.325 espécimes distribuídos em 25 espécies e três famílias, utilizando quatro métodos de coleta: rede entomológica, guardachuva entomológico, pitfall e malaise em 12 meses de coleta. Assim pode-se inferir que o método apresentado neste trabalho foi eficiente, pois coletou, 969 ortópteros distribuídos em 24 espécies, 19 gêneros, e três famílias em cinco meses de coletas.

Apesar dos poucos parâmetros existentes para comparar a eficiência de métodos de coleta de acridóideos e proscopideos em geral, os resultados apresentados neste trabalho mostraram eficiência do método proposto neste estudo para a coleta de insetos destes grupos. O número de amostras variou de cinco a sete dependendo da variável resposta (ninfa, adulto ou total), e a distancia percorrida de $25 \mathrm{~m}$ aliada ao número de sete amostras mostrou-se ideal para coleta de acridóideos.

Devido ao reduzido número de trabalhos envolvendo estudos sobre tamanho ideal de amostra para coleta de acridoídeos ressalta-se a importância deste estudo, onde os resultados apresentados podem servir de base para futuros trabalhos com este grupo de insetos, aperfeiçoando este método e a forma de amostragem.

\section{REFERÊNCIAS}

Carvalho, N.L. \& E.C. Costa, 2010. Análise faunística de gafanhotos (Orthoptera, Acridoidea: Acrididae, romaleidae e proscopiidae), no município de São Sepé, RS, Dissertação (mestrado) - Universidade Federal de Santa Maria, Centro de Ciências Rurais, Programa de Pós-Graduação em Agronomia.

Confalonieri, R.A., 2004. Jackknife-derived visual approach for sample size determination. Rivista Italiana di Agrometeorologia, 1: 9-13.

Fernandes, M.G., A.C. Busoli \& J.C. Barbosa, 2002. Amostragem seqüencial de Spodoptera frugiperda (J. E. Smith, 1797) Lepidoptera, Noctuidae) em algodoeiro. Revista brasileira de Agrociência, 8: 213-218.

Fonseca, J.S. \& G.A. Martins, 1996. Curso de estatística. 6.ed. São Paulo: Atlas, $320 \mathrm{p}$.

Hagstrum, D.W., P.W. Flinn \& D. Shuman, 1994. Acoustical monitoring of stored-grain insects: an automated system. In: Proceedings of the International Working Conference on Stored-Product Protection, 6, 17-21 April, Canberra, Australia p. 403-405.

Lutinski, C.J., F.R.M. Garcia, M.K.M. Costa \& J.A. Lutinski, 2009. Flutuação populacional de gafanhotos na Floresta Nacional de Chapecó, Santa Catarina. Ciência Rural, 39: 555-558.

Manly, B.F.J., 1997. Randomization, bootstrape and Monte Carlo methods in biology. $2^{\text {nd }}$ ed. New Zealand: University of Otaga, $356 \mathrm{p}$.

Moreno, J.A., 1961. Clima do Rio Grande do Sul. Porto Alegre: Secretaria da Agricultura, $73 \mathrm{p}$.

Pedigo, L.P., 1994. Introduction to sampling arthropod populations. p. 1-11. In: Pedigo, L.P. \& G.D. Buntin (Eds.). Handbook of sampling methods for arthropods in agriculture, Boca Raton: CRC Press, 714 p.

Richman, D.B., D.C. Lightfoot, C.A. Sutherland \& D.J. Ferguson, 1993. A manual of the grasshoppers of New Mexico Orthoptera: Acrididae and Romaleidae. Las Cruces, NM. México: New Mexico State University Cooperative Extension Service.

Subramanyam, B.H. \& D.W. Hagstrum, 1996. Sampling, p. 135193 In: Subramanyam, B. \& D.W. Hagstrum (Eds.) Integrated management of Insects in stored products. New York: M. Dekker, 426p.

Recebido em: 25/11/2012

Aceito em: 22/04/2013 
Como citar este artigo:

N.L. Carvalho, E.C. Costa, D.B. Souza \& J. Garlet, 2013. Tamanho e Número Ideal de Amostras para Coleta de Gafanhotos na Região Depressão Central do Rio Grande do Sul. EntomoBrasilis, 6(2): 119-125.

Acessível em: http://www.periodico.ebras.bio.br/ojs/index.php/ebras/article/view/294. doi:10.12741/ebrasilis.v6i2.294
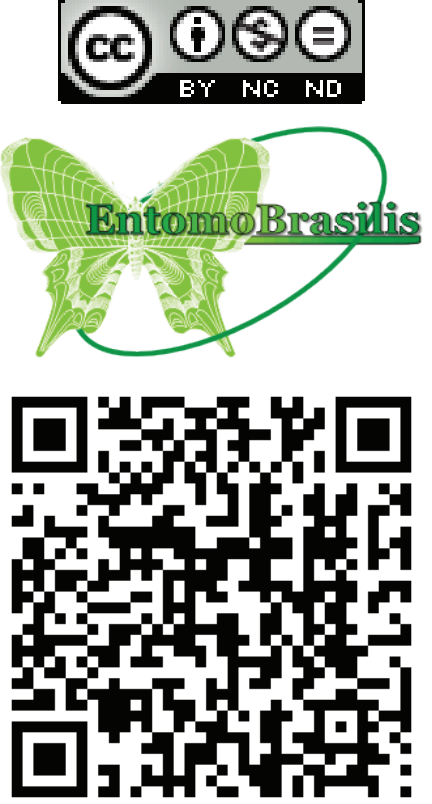